Virginia Commonwealth University VCU Scholars Compass

2017

\title{
Laser-Induced Breakdown Spectroscopy (LIBS) in a Novel Molten Salt Aerosol System
}

\author{
Ammon N. Williams \\ Supathorn Phongikaroon \\ Virginia Commonwealth University
}

Virginia Commonwealth University, williamsan25@vcu.edu

Follow this and additional works at: http://scholarscompass.vcu.edu/egmn_pubs

Part of the Mechanical Engineering Commons, and the Nuclear Engineering Commons

(C) The Author(s) 2016 Reprints and permissions: sagepub.co.uk/journalsPermissions.nav

\section{Downloaded from}

http://scholarscompass.vcu.edu/egmn_pubs/37 


\author{
Ammon N. Williams and Supathorn Phongikaroon
}

\begin{abstract}
In the pyrochemical separation of used nuclear fuel (UNF), fission product, rare earth, and actinide chlorides accumulate in the molten salt electrolyte over time. Measuring this salt composition in near real-time is advantageous for operational efficiency, material accountability, and nuclear safeguards. Laser-induced breakdown spectroscopy (LIBS) has been proposed and demonstrated as a potential analytical approach for molten $\mathrm{LiCl}-\mathrm{KCl}$ salts. However, all the studies conducted to date have used a static surface approach which can lead to issues with splashing, low repeatability, and poor sample homogeneity. In this initial study, a novel molten salt aerosol approach has been developed and explored to measure the composition of the salt via LIBS. The functionality of the system has been demonstrated as well as a basic optimization of the laser energy and nebulizer gas pressure used. Initial results have shown that this molten salt aerosolLIBS system has a great potential as an analytical technique for measuring the molten salt electrolyte used in this UNF reprocessing technology.
\end{abstract}

\title{
Keywords
}

Laser-induced breakdown spectroscopy, nuclear safeguards, pyroprocessing, molten salts

Date received: 24 November 2015; accepted: I March 2016

\section{Introduction}

Pyroprocessing technology is a dry reprocessing alternative to traditional aqueous methods.' In this process, the uranium in the used nuclear fuel (UNF) is electrochemically dissolved at an anode and transported through a molten salt electrolyte to a cathode inside an electrorefiner (ER). As part of the process chemistry, fission products, rare earth elements, and transuranics (including plutonium) accumulate in the molten salt. Monitoring the composition of the molten salt within the ER is important from operation efficiency, material accountability, and nuclear safeguards perspectives. ${ }^{2}$ However, analytical methods to measure the salt have been limited due to a high temperature operating condition (typically, 723-773 K) and high radiation environment that exist within the process. As a result, salt samples are usually drawn and measured via inductively coupled plasma mass spectrometry (ICP-MS). ${ }^{3}$ This process of extraction, material transfer, and later sample preparation is cumbersome and can impose significant delay between sampling and compositional results.

Laser-induced breakdown spectroscopy (LIBS) has been proposed as an alternative analytical approach to ICP-MS since it can be done in situ, with little to no sample preparation, and compositional information of the sample can be obtained in near real-time. ${ }^{4}$ In LIBS, a pulsed laser is focused onto a material surface to vaporize and ionize the sample in the creation of a plasma plume. As the plasma cools, the ionized atoms return to ground states with the emission of characteristic light which can be collected via optics. From the spectrum, qualitative and quantitate information about the sample composition can be obtained. Effenberger was among the first to demonstrate LIBS analysis in a molten $\mathrm{LiCl}-\mathrm{KCl}$ salt by using a static surface approach, ${ }^{5}$ meaning that the laser light was focused downwards onto the top surface of the melted salt in a crucible. Here, $\mathrm{CrCl}_{3}, \mathrm{CoCl}_{2}$, and $\mathrm{MnCl}_{2}$ were studied in the molten salt matrix at $773 \mathrm{~K}$. This work showed that LIBS analysis in the molten salt was feasible. Using the static surface configuration similar to Effenberger, ${ }^{5}$ Hanson et al. performed

Department of Mechanical and Nuclear Engineering, Virginia Commonwealth University, Richmond, VA, USA

\section{Corresponding author:}

Ammon N. Williams, Department of Mechanical and Nuclear Engineering, Virginia Commonwealth University, 40I West Main Street, Richmond, VA 23284, USA.

Email: williamsan25@vcu.edu 
an in-depth LIBS study of $\mathrm{CeCl}_{3}$ and $\mathrm{MnCl}_{2}$ in $\mathrm{LiCl}-\mathrm{KCl}$ salts at temperatures ranging from $523 \mathrm{~K}$ (solid) to $773 \mathrm{~K}$ (liquid). ${ }^{6}$ Results of the study showed that, in general, the molten samples had less self-absorption and lower relative standard deviations (\%RSDs) than the solid samples. Weisberg et al. also studied a molten $\mathrm{LiCl}-\mathrm{KCl}$ salt with additives of $\mathrm{EuCl}_{3}$ and $\mathrm{PrCl}_{3}$ using the static surface approach. ${ }^{7}$ In this work, a thin film was observed on the top surface of the salts containing $\mathrm{Eu}$ and $\mathrm{Pr}$ but not on blank $\mathrm{LiCl}-\mathrm{KCl}$ samples. The composition of the film with respect to the bulk was not reported; however, the presence of the film influenced the shot-to-shot results since plasma formed on the liquid surface differed from those formed on the solid film surface. To address this issue, the authors used conditional analysis to eliminate large shot-to-shot fluctuations within the collected spectra, thus effectively grouping shots that fell only on the liquid surface. In addition to the conditional analysis, a partial least squares (PLS) method was used to obtain qualitative and quantitative results. More recently, Smith et al. from the Argonne National Laboratory have designed a probe that can be used to remotely analyze the static surface of the molten salt within a glovebox environment. ${ }^{8}$ To date, feasibility studies have been done to measure $U, P u$, and $\mathrm{Np}$ in the $\mathrm{LiCl}-\mathrm{KCl}$ salt.

These aforementioned LIBS studies conducted in a molten $\mathrm{LiCl}-\mathrm{KCl}$ salt have used a static surface approach. Whereas this approach is feasible and can yield compositional information of the salt, many potential issues exist. For example, in the formation of the plasma on a liquid sample, a shock wave is created which tends to splash the sample-potentially causing a quenching of the plasma and/ or lens damage and degradation. In addition, liquid quenching of the plasma can further reduce signal and the plasma lifetime. ${ }^{9}$ Other potential issues are the film formation/dross layer and bubble formation on the top surface of the salt, which can lead to homogeneity concerns. These aspects provide a motivation for the development of a new innovative LIBS sampling configuration for molten salt analysis.

One potential method is to use a LIBS-aerosol measurement technique. ${ }^{10}$ In this approach, the laser is focused within an aerosol gas stream to create the LIBS plasma. This technique is often used for online monitoring of environmental air samples for toxic metals. ${ }^{1-13}$ In these cases, the aerosol concentration within the gas stream is low, leading to high shot-to-shot variation as a result of low probability of particle interaction with the plasma. Diwakar et al. have shown that the plasma-particle interaction can be defined by Poisson modeling statistics. ${ }^{14}$ As a result, this supports the conditional analysis technique for excluding shots below a certain threshold.

In many cases, the aerosol is generated from a liquid sample via an aerosol generator. Here, the aerosol concentration within the gas stream can be sufficiently high as to reduce the probability of null shots; however, significant shot-to-shot variation is still observed. Huang et al. observed that the shot-to-shot variation could be large due to fluctuations in the number of aerosol particles consumed per plasma volume. ${ }^{15,16}$ Poulain and Alexander, ${ }^{17}$ as well as Schechter, ${ }^{18}$ observed the large shot-to-shot variation in liquid aerosols and concluded that the variation can be attributed to (I) the number of particles within the plasma volume, (2) the location and size of the particles within the plasma, and (3) liquid droplet interference with the incoming laser light. As a result, Schechter developed a basic algorithm to perform conditional analysis on the collected spectra to reduce variation prior to ensemble averaging. ${ }^{18}$ Using the above conditional analysis scheme, Schechter was able to significantly improve the sensitivity of the system.

Despite challenges with particle-plasma interactions and interference, LIBS analysis from liquid aerosols has been shown to perform well. Kumar et al. used a Meinhard nebulizer to generate an aerosol and found that the aerosol approach yielded better results than a liquid jet method. ${ }^{19}$ They concluded that this in part was due to better utilization of the laser energy to ionize the sample in the aerosol stream, whereas much of the laser energy was used to first vaporize the sample in the liquid jet case. Martin and Cheng measured chromium in water and reported a limit of detection for $\mathrm{Na}$ down to $400 \mathrm{ng}$ per dry standard cubic meter $(\mathrm{dscm}){ }^{20}$ Cahoon and Almirall measured the concentrations of $\mathrm{Sr}, \mathrm{Ba}, \mathrm{Mg}$, and $\mathrm{Ca}$ in water using both an aerosol and micro-droplet approach. ${ }^{9}$ Here, both methods resulted in ultralow detection limits with a \%RSD of the order of $1 \%$. Alvarez-Trujillo et al. performed stand-off LIBS in a liquid aerosol and determined that they could detect $\mathrm{Na}$ down to $55 \mathrm{ppm}$ in water at a distance of $10 \mathrm{~m} .^{21}$

In addition to a good performance, an aerosol approach has several advantages to a static surface configuration with regards to an online measuring system for molten salts. In an aerosol sampling approach, the liquid sample can be drawn continuously from the bulk of the salt, thus reducing variation due to inhomogeneity. In addition, since splashing, bubble formation, and surface perturbations are not an issue with the aerosol approach, the shot-to-shot frequency of the sampling system is less limited. Lastly, the laser energy is better utilized to ionize the sample. Due to these advantages, it has been proposed to use this approach to measure molten salt. To date, the high temperature aerosol generation of molten salts has never been reported. As a result, a novel molten salt aerosol and LIBS system is being developed (patent pending) and has been shown to have a promising potential for online measuring of the molten salt composition. In this system, argon gas is used to create aerosol in high temperature (773 K) salt using a Collison nebulizer. The aerosol is introduced into a sampling chamber where the laser light and plasma emissions are transmitted in and out via quartz ports. Following the sampling chamber, the aerosol sample is 


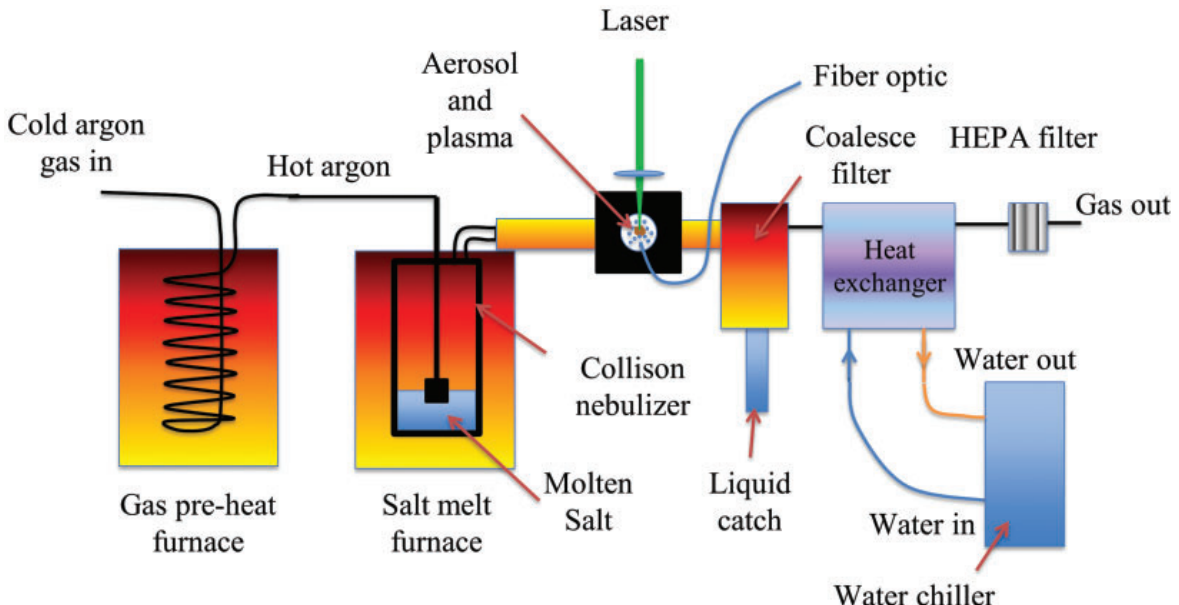

Figure I. Illustration of the proposed aerosol-LIBS setup.

passed through a coalesce filter to remove the molten salt droplets prior to cooling the gas stream to room temperature. Currently, the system has been tested and optimization of the laser energy and gas pressure will be initially reported here-illustrating its promising potential in composition detection in a molten salt system for pyroprocessing technology.

\section{Setup and Method}

An illustration of the molten salt aerosol-LIBS configuration using a Collison nebulizer is shown in Figure I. In the first step of the process, cold argon gas is heated to $773 \mathrm{~K}$ before entering the Collison nebulizer to prevent salt freezing. This is done using $4.3 \mathrm{~m}$ of coiled 0.25 inch diameter stainless steel tubing within a Kerr Lab furnace. The hot gas then enters a three-jet Collison nebulizer purchased from BGI, Inc., to generate the molten salt aerosol. The nebulizer was modified in order to operate safely up to $773 \mathrm{~K}$. The flow of argon and molten salt droplets then passes into a sampling chamber with three quartz ports from Rayotek, Inc. Through the top port, the laser light is focused into the aerosol stream to create the plasma. Through the back port, the plasma light is collected via a fiber optic cable and optics positioned outside the sampling chamber. The front port is used for visualization of the plasma. The sampling chamber was designed and built in house to accommodate up to 100 psi. The aerosol stream then passes into a coalesce filter (with high temperature seals) from United Filtration Systems, Inc., which removes the aerosol from the gas stream. The entire system was fitted together using Swagelok and NPT fittings with a high temperature thread sealant design. The sampling chamber and coalesce filter were heated via two $125 \mathrm{~W}$ cartridge heaters. The hot gas exiting the coalesce filter then passes through a heat exchanger to cool it down to ambient temperatures before being passed through a HEPA filter and vented into the exhaust system.

The inert atmosphere glovebox and the experimental setup are shown in Figure $2 a$ and $2 b$, respectively. Here, a Q-smart $450 \mathrm{Nd}$ :YAG laser (Quantel USA, Inc.) was mounted vertically on the outside of the glovebox and the laser light was directed via optics into the glovebox through a quartz window located in the glovebox wall. A laser mirror within the glovebox was used to guide the laser into the sampling chamber through a lens with a $75 \mathrm{~mm}$ focal length $(f)$. The focused laser light created a plasma in the center of the sampling chamber. Light from the plasma was collected via an $f=75 \mathrm{~mm}$ lens and then focused ( $f=100 \mathrm{~mm}$ lens) into a $4 \mathrm{~m}$ long fiber optic cable from Ocean Optics, Inc. The fiber optic cable carried the light to a Michelle 5000 spectrometer and iStar ICCD detector located outside of the glovebox. The Michelle spectrometer and ICCD detector were purchased from Andor Technologies, Inc.

Salt samples were made using $\mathrm{CeCl}_{3}$ (99.99\%), $\mathrm{LiCl}$ (99.99\%), and $\mathrm{KCl}$ (99.95\%) purchased from Alfa Aesar. The $\mathrm{LiCl}$ and $\mathrm{KCl}$ salts were mixed at the eutectic ratio (44 wt \% $\mathrm{LiCl}$ and $56 \mathrm{wt} \% \mathrm{KCl}$ ) and the $\mathrm{CeCl}_{3}$ composition was set at $5 \mathrm{wt} \%$ for this study. The salts were formed into ingots by mixing them in an inert atmosphere glovebox, drying at $573 \mathrm{~K}$ for $24 \mathrm{~h}$, and then pre-melting for $12 \mathrm{~h}$ before rapidly freezing them into solid slugs. Each ingot contained approximately $4 \mathrm{l} .5 \mathrm{~g}$ of total salt. For each experiment, a single salt ingot was loaded into the Collison nebulizer jar and then the system was ramped up to $773 \mathrm{~K}$ at approximately $4 \mathrm{~K} / \mathrm{min}$. The system was held at temperature for $8 \mathrm{~h}$ prior to operating the system to ensure that the sample was fully melted and homogeneous. Following melting, argon gas at the desired pressure was introduced into the nebulizer to create the aerosol. The Collison nebulizer uses the Venturi effect to draw up 


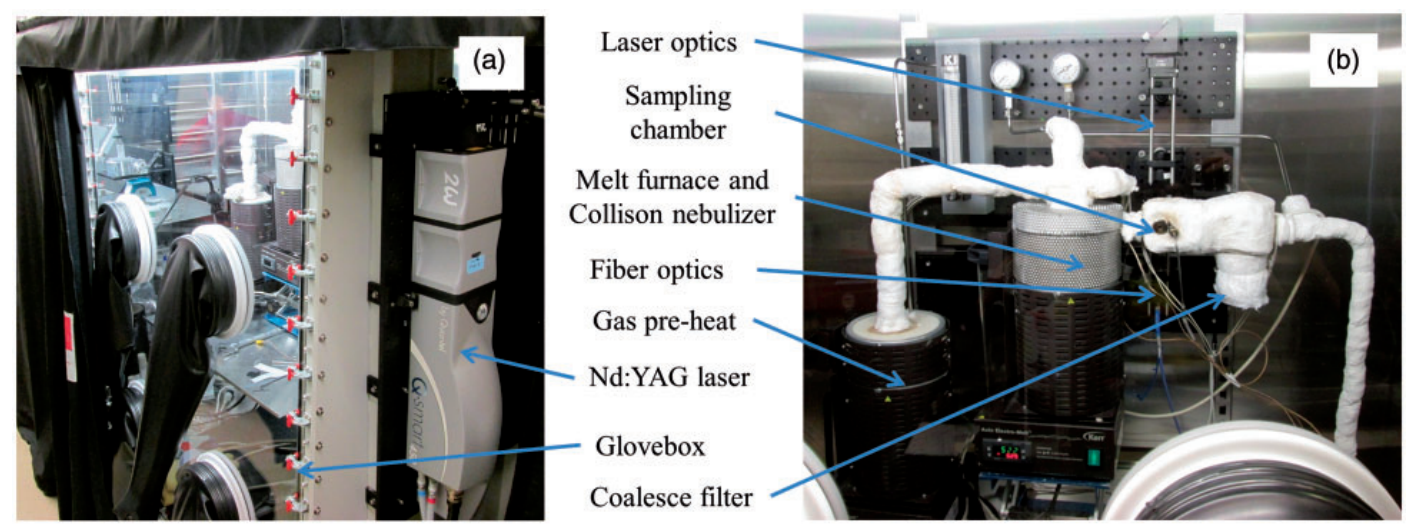

Figure 2. (a) Picture of the inert atmosphere glovebox with laser mounting and safety curtains. (b) Close up of the aerosol-LIBS system mounted within the glovebox.

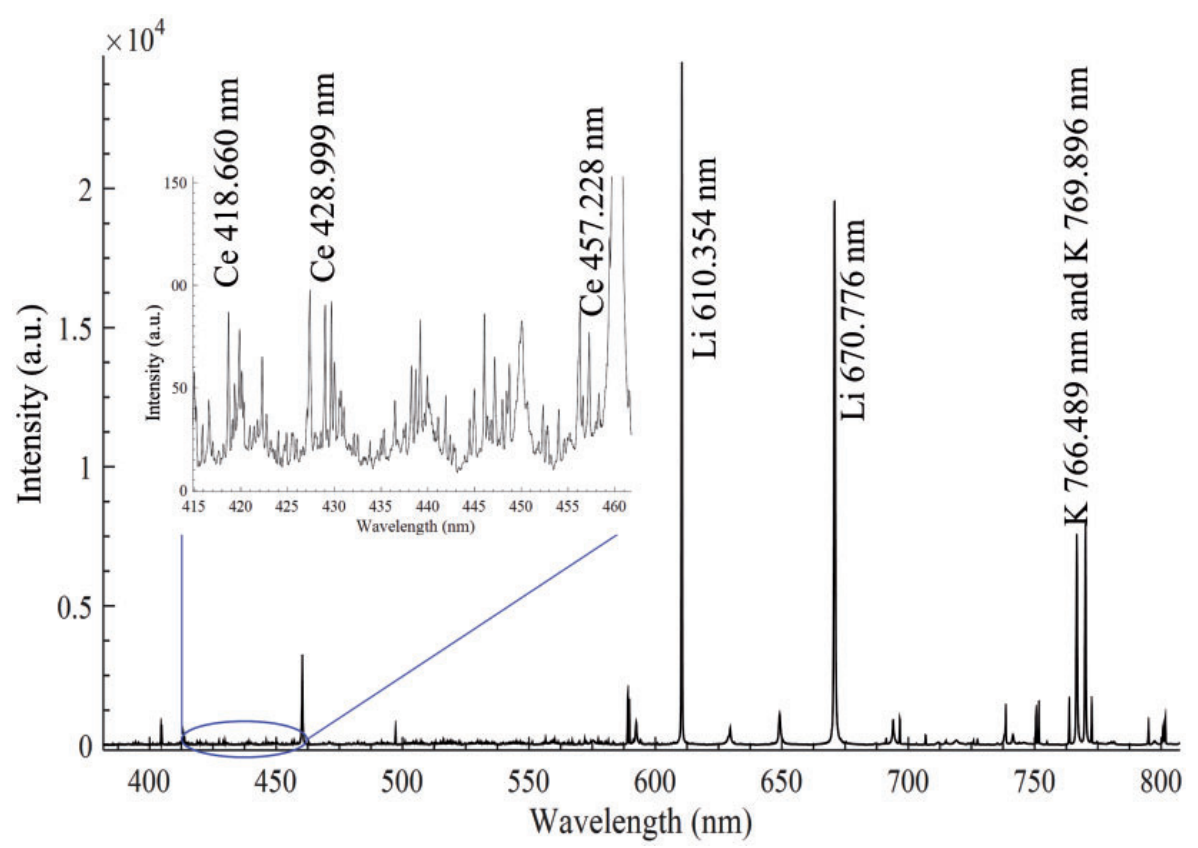

Figure 3. A representative LIBS spectrum obtained from the $5 \mathrm{wt} \% \mathrm{CeCl}_{3}-\mathrm{LiCl}-\mathrm{KCl}$ samples.

and cycle the sample within the nebulizer jar. As a result of the long melting period and forced convection during operation of the nebulizer, it is assumed that the salt composition in the aerosol stream was the same as the bulk salt composition.

Initial experiments were done to explore the effect of the nebulizer pressure and laser energy on the signal-tonoise ratio (SNR). Here, the SNR is the peak intensity divided by the background intensity near the peak of interest. The laser energy was varied between $40 \mathrm{~mJ}$ and $140 \mathrm{~mJ}$ and the nebulizer pressure was varied between 10 psi and $60 \mathrm{psi}$. For each variation, three repetitions were completed, each comprising of 250 laser shots. The 250 spectra from each repetition were averaged to provide a single representative spectrum.

\section{Results and Discussion}

For each element in the sample, the strongest expected peaks were determined using the NIST database for atomic spectral lines. ${ }^{22}$ Three cerium peaks were selected: $418.660 \mathrm{~nm}$ (the dominant peak), $428.994 \mathrm{~nm}$ and 457.228 lines. These lines were picked due to high intensity and/or low interference with other peaks. For $\mathrm{Li}$, there are a number of strong peaks; most notably are the lines at $460.289 \mathrm{~nm}, 610.354 \mathrm{~nm}$, and $670.776 \mathrm{~nm}$. For $\mathrm{K}$, there are two strong lines at $766.489 \mathrm{~nm}$ and $769.896 \mathrm{~nm}$. A representative spectrum for the $5 \mathrm{wt} \% \mathrm{CeCl}_{3}-\mathrm{LiCl}-\mathrm{KCl}$ salt obtained via the aerosol-LIBS system is shown in Figure 3.

Figure 4 shows the SNR for the $418.660 \mathrm{~nm}$, $428.999 \mathrm{~nm}$, and $457.228 \mathrm{~nm}$ lines as a function of the 


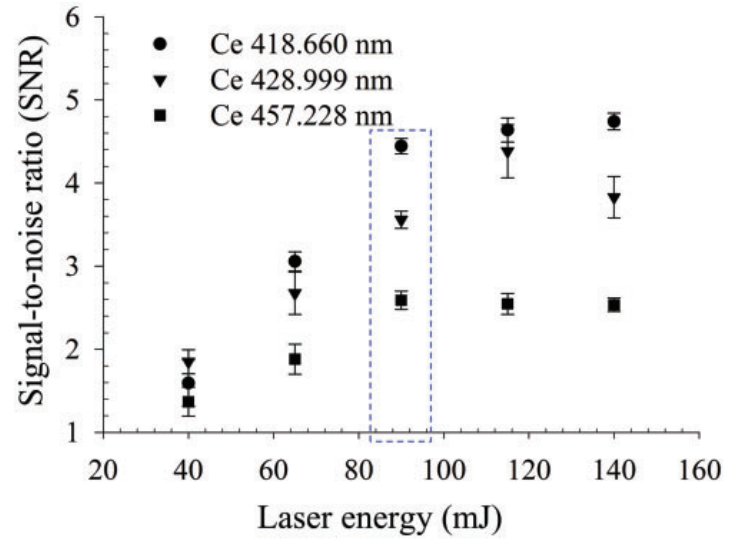

Figure 4. The SNR as a function of the of the laser energy.

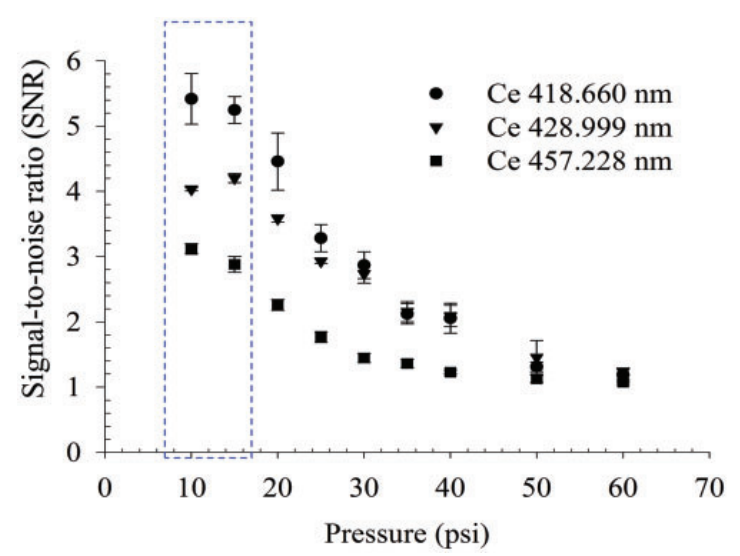

Figure 5. SNR as a function of the argon gas pressure in a threejet Collison nebulizer.

laser energy used. The results show that the SNR increases linearly up to about $90 \mathrm{~mJ}$ where the trend flattens off. A similar plateau was observed by Carranza and Hahn. ${ }^{13}$ In most cases, the goal is to maximize the SNR; however, in order to operate at a safer, less energy intensive range for the laser, a different approach was used. In this case, the intersection between the upward slope and the plateau was identified as the optimal value since it provides the lowest laser energy while still achieving high SNRs.

Results from the nebulizer pressure variation are shown in Figure 5 and it can be seen that the LIBS signal decreases significantly with increased pressure. As the gas pressure increases, the flow rate of the aerosol-gas stream increases and different conditions within the sampling chamber exist. Due to the location, complexity, and extreme temperature of the system, it was not possible to measure the molten salt aerosol concentration and size distribution with respect to operation pressure. From the literature on the Collison nebulizer, it is anticipated that the size distribution of the particles will not change strongly as a function of pressure. ${ }^{23}$ However, as the pressure increases, the gas flow rate increases and the overall salt concentration would decrease. ${ }^{23}$ As a result, the total salt mass per plasma volume is likely reduced at increased pressures and the signal becomes dominated by the argon gas background. Larger SNR are then achieved at the lower operating pressure due to an increase in the aerosol concentration. However, it is anticipated that with further reduced pressure, the aerosol concentration could increase to a point where particle interference with the laser would become dominant and the SNR would decrease. Below approximately $10 \mathrm{psi}$, the nebulizer flow becomes unstable and no further testing was performed. As a result, a gas operating pressure of 10-15 psi appears to be optimal in order to maximize the SNR. In a future study, the gate delay, calibration curves, and limits of detection will be explored and presented for this system.

\section{Conclusion}

A novel molten salt aerosol-LIBS system has been proposed, designed, and initially tested for a potential method for on-line measuring of molten salt composition in the pyroprocessing of UNF. The system utilizes a Collison nebulizer to generate the molten salt aerosol within an argon gas stream. Initial testing has demonstrated that the system provides atomic spectral information of the elements within the salt. The optimal values for the laser energy and nebulizer gas pressure were determined to be $90 \mathrm{~mJ}$ and 10-15 psi, respectively. Future studies will further explore system parameters and optimization. This preliminary study has shown that this novel molten salt aerosol-LIBS system has a great potential for future use as an alternative for monitoring special nuclear materials in molten salt for nuclear material accountability and safeguards concerns.

\section{Acknowledgments}

The authors would like to thank Dalsung Yoon for his assistance with this project.

\section{Conflict of Interest}

None declared

\section{Funding}

This work was supported using funding received from the DOE Office of Nuclear Energy's Nuclear Energy University Programs (NEUP), work package \# NU-I2-VA-VCU-0203-0I. Additional financial support was provided by the Nuclear Regulatory Commission Fellowship.

\section{References}

I. J.J. Laidler, J.E. Battles, W.E. Miller, J.P. Ackerman, E.L. Carls. "Development of Pyroprocessing Technologies". Prog. Nucl. Energy. |997. 3I(I-2): |3|-|40. 
2. H.E. Garcia, M.J. Lineberry, S.E. Aumeier, H.F. McFarlane. "Proliferation Resistance of Advanced Sustainable Nuclear Fuel Cycles”. Nucl. Plant J. 2002. 20(I): I8-27.

3. S. Phongikaroon, M. Simpson. "Equilibrium Model for lon Exchange between Multivalent Cations and Zeolite-A in a Molten Salt". AIChE J. 2006. 52(5): 1736-1743.

4. C. Pasquini, J. Cortez, L.M.C. Silva, F.B. Gonzaga. "Laser Induced Breakdown Spectroscopy". J. Braz. Chem. Soc. 2007. 18(3): 463-5I2.

5. A.J. Effenberger. Methods for Measurement of Heterogeneous Materials with Laser-Induced Breakdown Spectroscopy (LIBS). [Ph.D. Dissertation]. San Diego: University of California, 2009.

6. C. Hanson, S. Phongikaroon, J.R. Scott. "Temperature Effect on LaserInduced Breakdown Spectroscopy Spectra of Molten and Solid Salts". Spectrochim. Acta, Part B. 2014. 97: 79-85.

7. A. Weisberg, R.E. Lakis, M.F. Simpson, L. Horowitz, J. Craparo. "Measuring Lanthanide Concentrations in Molten Salt using LaserInduced Breakdown Spectroscopy (LIBS)”. Appl. Spectrosc. 2014. 68(9): 937-948

8. N.A. Smith, J.A. Savina, M.A. Williamson. "Application of Laser Induced Breakdown Spectroscopy to Electrochemical Process Monitoring of Molten Chloride Salts". Poster presented at: International Atomic Energy Agency (IAEA) Symposium on International Safeguards 20I4. Vienna, Austria; October 20-24, 2014

9. E.M. Cahoon, J.R. Almirall. "Quantitative Analysis of Liquids from Aerosol and Microdrops using Laser Induced Breakdown Spectroscopy”. Anal. Chem. 2012. 84(5): 2239-2244.

I0. M.Z. Martin, M. Cheng, R.C. Martin. "Aerosol Measurement by LaserInduced Breakdown Spectroscopy: A Review”. Aerosol Sci. Technol. 1999. 31(6): 409-421.

II. B.T. Fisher, H.A. Johnson, S.G. Buckley, D.W. Hahn. "Temporal Gating for the Optimization of Laser-Induced Breakdown Spectroscopy Detection and Analysis of Toxic Metals". Appl. Spectrosc. 200I. 55(10): 1312-1319.

I2. J.E. Carranza, B.T. Fisher, G.D. Yoder, D.W. Hahn. "On-line Analysis of Ambient Air Aerosols using Laser-Induced Breakdown Spectroscopy”. Spectrochim. Acta, Part B. 200I. 56(6): 85I-864.
13. J.E. Carranza, D.W. Hahn. "Sampling Statistics and Considerations for Single-Shot Analysis using Laser-Induced Breakdown Spectroscopy". Spectrochim. Acta, Part B. 2002. 57(6): 779-790.

I4. P.K. Diwakar, K.H. Looper, A.-M. Matiaske, D.W. Hahn. "Laser-Induced Breakdown Spectroscopy for Analysis of Micro and Nanoparticles". J. Anal. At. Spectrom. 2012. 27: III0-III9.

I5. J.S. Huang, C.-B. Ke, L.-S. Huang, K.-C. Lin. "The Correlation between Ion Production and Emission Intensity in the Laser-Induced Breakdown Spectroscopy of Liquid Droplets". Spectrochim. Acta, Part B. 2002. 57(I): 35-48.

16. J. Huang, C. Ke, K. Lin. "Matrix Effect on Emission/Current Correlated Analysis in Laser-Induced Breakdown Spectroscopy of Liquid Droplets". Spectrochim. Acta, Part B. 2004. 59(3): 32I-326.

I7. D.E. Poulain, D.R. Alexander. "Influences on Concentration Measurements of Liquid Aerosols by Laser-Induced Breakdown Spectroscopy”. Appl. Spectrosc. 1995. 49(5): 569-579.

18. I. Schechter. "Direct Aerosol Analysis by Time Resolved Laser Plasma Spectroscopy - Improvement by Single Shot Measurements". Anal. Sci. Technol. 1995. 8(4): 779-786.

19. A. Kumar, F.Y. Yueh, T. Miller, J.P. Singh. "Detection of Trace Elements in Liquids by Laser-Induced Breakdown Spectroscopy with a Meinhard Nebulizer". Appl. Opt. 2003. 42: 6040-6046.

20. M. Martin, M. Cheng. "Detection of Chromium Aerosol Using TimeResolved Laser-Induced Plasma Spectroscopy”. Appl. Spectrosc. 2000. 54(9): 1279-1285.

21. L.A. Alvarez-Trujillo, A. Ferrero, J. Javier Laserna. "Preliminary Studies on Stand-Off Laser-Induced Breakdown Spectroscopy Detection in Aerosols”. J. Anal. At. Spectrom. 2008. 23: 885-888.

22. A. Kramida, Yu. Ralchenko, J. Reader, NIST ASD Team. "NIST Atomic Spectra Database (Version 5.3)". National Institute of Standards and Technology. http://www.nist.gov/pml/data/asd.cfm [accessed Aug I5 2016]..

23. K.R. May. "The Collison Nebulizer: Description, Performance and Application”. J. Aerosol Sci. 1973. 4(3): 235-243. 